Materials Research Bulletin, Vol.29, No. 12, pp. 1255-1262, 1994

https://doi.org/10.1016/0025-5408(94)90149-X

\title{
SYNTHESE DES HYDROXYDES DE COBALT, NICKEL ET CADMIUM, PAR OXYDATION DES METAUX PAR L'OXYGENE EN PRESENCE D'AMMONIAQUE
}

\author{
A. LECERF et Y. CUDENNEC \\ Laboratoire de chimie des matériaux inorganiques et de cristallographie \\ Institut National des Sciences Appliquées \\ 20, avenue des Buttes de Coësmes \\ 35043 RENNES CEDEX (France)
}

(Received September 7, 1994; Communicated by E.F. Bertaut)

\begin{abstract}
Pure hydroxides of cobalt, nickel and cadmium have been synthesized by a new route. The powdered metals have been oxidized by dioxygen in aqueous ammonia. The reactions have been carried out at ambient temperature and pressure. Cobalt hydroxide $\mathrm{Co}(\mathrm{OH})_{2}$ is slowly oxidized to $\mathrm{CoOOH}$ by subsequent exposure to dioxygen. The $\mathrm{Ni}(\mathrm{OH}) 2$ synthesis is easier if nickel metal is activated by exposure to dihydrogen at about $300^{\circ} \mathrm{C}$.
\end{abstract}

MATERIALS INDEX: Hydroxide; cobalt; nickel; cadmium.

\section{Introduction}

On sait depuis le $19^{\text {ème }}$ siècle que le cobalt et le nickel sont solubles dans l'ammoniaque en présence d'air (1). Dès 1848 Nicklès a préparé l'hydroxyde de cadmium en plongeant une lame de métal dans l'ammoniaque au contact de la tournure de fer (2).

Les études entreprises par les électrochimistes montrent qu'il se forme un complexe amminé (3). La réaction mise en jeu peut s'écrire pour un métal divalent:

$$
\text { (1) } \mathrm{M}+1 / 2 \mathrm{O}_{2}+\mathrm{H}_{2} \mathrm{O}+\mathrm{nNH}_{3} \longrightarrow \mathrm{M}\left(\mathrm{NH}_{3}\right)_{\mathrm{n}}^{2+}+2 \mathrm{OH}^{-}
$$

Ou M désigne, le cobalt, le nickel ou le cadmium. Cette réaction s'accompagne d'une augmentation $\mathrm{du} \mathrm{pH}$ de la solution. En opérant dans des conditions où l'avancement de la réaction est suffisant, on peut espérer atteindre un $\mathrm{pH}$ assez élevé pour que l'hydroxyde $\mathrm{M}(\mathrm{OH})_{2}$ précipite selon la réaction: 


$$
\mathrm{M}\left(\mathrm{NH}_{3}\right)_{\mathrm{n}}{ }^{2+}+2 \mathrm{OH}^{-} \quad \triangleright \mathrm{M}(\mathrm{OH})_{2 \mathrm{~s}}+\mathrm{n} \mathrm{NH}_{3}
$$

Dans la mesure où la réaction (1) est possible, elle présente un très grand intérêt. En effet l'utilisation d'ammoniaque pure et d'oxygène pur doit permettre de préparer des hydroxydes de grande pureté, exempts des anions et cations parasites, inévitablement entraînés dans les réactions de précipitation habituellement utilisées pour la préparation de ces hydroxydes.

Il ne semble pas que cette réaction d'oxydation en présence d'ammoniaque ait été systématiquement utilisée pour la synthèse des hydroxydes métalliques. I1 existe toutefois des brevets qui revendiquent la préparation d'hydroxydes de cobalt et de nickel par oxydation du métal par l'air en présence d'eau. Concernant le cobalt le brevet est très ancien et il ne nous a pas été possible de nous le procurer. Un brevet Inco très récent (4) revendique un nouveau procédé de synthèse de $\mathrm{Ni}(\mathrm{OH})_{2}$ par oxydation du métal en présence d'eau, dans un intervalle de température compris ente $50^{\circ} \mathrm{C}$ et $210^{\circ} \mathrm{C}$, en présence d'un catalyseur. Le catalyseur peut être un acide minéral ou organique, un sel de nickel, un hydroxyde alcalin ou l'ammoniaque.

Par rapport aux autres solutés, l'ammoniac présente un double intérêt lorsque l'objectif est la synthèse d'un hydroxyde de grande pureté :

- C'est une molécule qui, à la différence des ions, n'est guère susceptible de s'intégrer au réseau cristallin des hydroxydes.

- C'est un gaz dans les conditions ordinaires de température et de pression, il sera donc facile de l'éliminer par un séchage sous vide de l'hydroxyde.

\section{Partie Expérimentale}

Synthèse: - Dans une première étape un appareillage très simple, représenté sur la figure.1, a été utilisé :

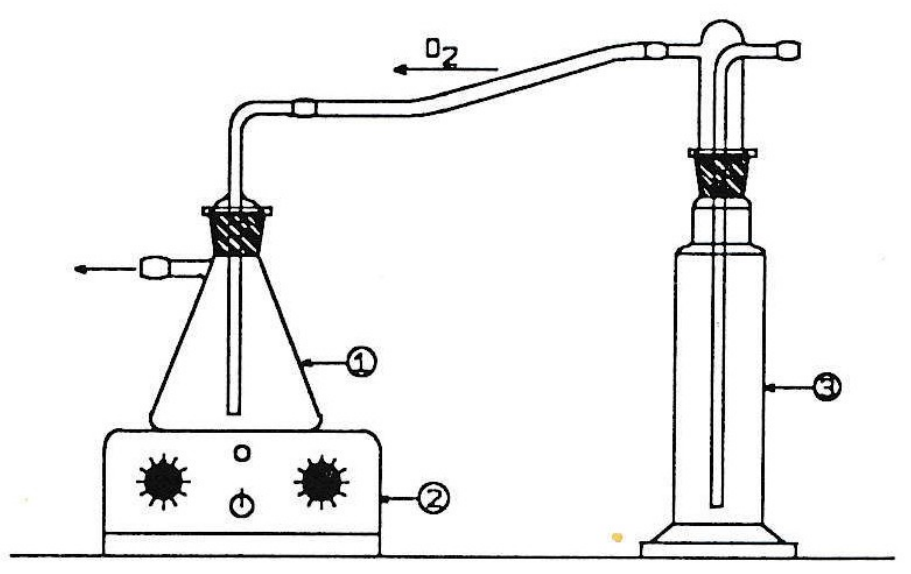

FIG.1

Mantage utıllsé pour la sunthèse

Le réacteur (1) est un erlen rodé muni d'un tube plongeur permettant de faire barboter l'oxygène dans la solution. La poudre métallique est introduite dans l'erlen, puis une solution commerciale d'ammoniaque à $32 \%$ est ajoutée. Le contenu du réacteur est agité par un agitateur magnétique (2). On fait passer un bulle à bulle d'oxygène dans la solution. L'oxygène passe préalablement dans un barboteur (3) contenant de l'ammoniaque à $32 \%$ o qui est renouvelée tous les jours. Ce dispositif permet d'éviter un déplacement rapide de l'ammoniac hors du réacteur par le courant gazeux. 
A titre d'exemple l'expérience réalisée avec le cobalt va être décrite: $20 \mathrm{~g}$ de cobalt MHO en poudre de qualité extra fine sont introduits dans le réacteur, on ajoute $300 \mathrm{~cm}^{3}$ d'ammoniaque (ammoniaque recta pur Prolabo à $32 \%$ ) puis on fait passer le courant d'oxygène sous agitation. La température n'est pas régulée mais est voisine de $20^{\circ} \mathrm{C}$.

On observe les phénomènes suivants :

- Après quelques heures une coloration rouge intense de la solution, caractéristique des complexes amminés du cobalt divalent.

- Après 24 heures, la présence d'hydroxyde de cobalt en suspension, le $\mathrm{pH}$ de la solution est alors très voisin de 14 . magnétique.

- Après 2 jours, la disparition du cobalt métallique aisément repérable sur le barreau $\mathrm{Co}(\mathrm{OH})_{2}$ est récupéré par filtration, lavé rapidement à l'eau distillée, séché sous vide dans un dessiccateur à potasse puis dans un dessiccateur à tamis moléculaire.

Dans l'expérience précédente on a remplacé le cobalt par du cadmium en billes (cadmium sphérique Wiaux) sans modifier les autres paramètres. On a obtenu dans ces conditions l'hydroxyde de cadmium $\beta-\mathrm{Cd}(\mathrm{OH})_{2}$.

La précipitation de $\mathrm{Ni}(\mathrm{OH})_{2}$ est plus difficile, $\mathrm{Ni}(\mathrm{OH})_{2}$ a pu être préparé selon le mode opératoire ci-dessus en 8 jours. On a toutefois observé que cette précipitation a un caractère aléatoire d'un essai à l'autre. Dans tous les cas on observe la formation du complexe amminé du nickel, caractérisé par sa couleur bleue intense, mais dans certains essais il n'y a pas précipitation de l'hydroxyde. Ces fluctuations ont été attribuées au dispositif expérimental utilisé qui ne permet pas un contrôle précis des conditions opératoires. Dans le but de contrôler avec précision ces conditions, un réacteur Buschi BEP 280 type I a été utilisé. Ce réacteur parfaitement étanche permet d'opérer à des pressions de gaz connues exactement et constantes. Un dispositif spécial d'agitation assure un brassage très efficace des gaz et de la solution. Une double enveloppe permet un réglage précis de la température par circulation d'un liquide thermostaté.

Caractérisation :- Les dosages des métaux et le nombre d'oxydation du cobalt ont été déterminés par volumétrie à partir, respectivement, d'une solution d' E.D.T.A. et d'une solution de sel de Mohr. Un chromatographe Dionex DX 100 équipé d'une colonne Ionpac AS4 ASC a été utilisé pour la recherche des anions éventuellement présents. Les diagrammes de diffraction X ont été enregistrés sur un diffractomètre Rigaku D Max II. Les clichés de microscopie électronique à balayage haute résolution ont été réalisés sur un microscope Geol JSM 6400 F. Un analyseur Adamel ATD 67 et une thermobalance Adamel ont été utilisés pour les thermolyses de l'hydroxyde de cobalt.

\section{Résultats et discussion}

\section{$\underline{\text { A - Hydroxyde de Cobalt }}$}

Plusieurs essais ont été réalisés en faisant varier la masse de cobalt utilisée et le temps de contact avec la solution sous courant d'oxygène. les résultats des analyses chimiques sont consignés dans le tableau I où $n$ désigne le nombre d'oxydation du cobalt.

\begin{tabular}{ccc}
\multicolumn{3}{c}{ Tableau I } \\
Durée de l'essai & Co $\%$ & $\mathrm{n}$ \\
2 jours & 62.5 & 2.08 \\
5 jours & 62.7 & 2.14 \\
12 jours & 62.8 & 2.37
\end{tabular}

Les taux de cobalt observés sont en bon accord avec le taux calculé de $63.40 \%$ pour $\mathrm{Co}(\mathrm{OH})_{2}$. Les dosages effectués par chromatographie ionique n'ont mis en évidence aucun anion, à une teneur 
détectable dans les conditions opératoires utilisées. La colonne 3 du tableau I met en évidence une oxydation progressive du cobalt lorsque $\mathrm{Co}(\mathrm{OH})_{2}$ reste en contact avec l'oxygène.

Le diagramme de diffraction X (fig.2) du solide le moins oxydé montre bien que $\mathrm{CoOOH}$ est déjà présent en très faible quantité. On observe que la teneur en $\mathrm{CoOOH}$ croît en même temps que le nombre d'oxydation. Par contre le taux de cobalt observé ne subit pas de variation significative, ce qui est en bon accord avec la présence de $\mathrm{CoOOH}$. En effet, le taux de cobalt de $64.10 \%$ dans $\mathrm{CoOOH}$ est très voisin de celui de $\mathrm{Co}(\mathrm{OH})_{2}$.

L'indexation des diagrammes de diffraction $\mathrm{X}$ a permis de calculer les paramètres de la maille hexagonale de $\mathrm{Co}(\mathrm{OH})_{2}$. On remarque que les valeurs obtenues sont indépendantes du nombre d'oxydation, comme on peut le prévoir pour un système diphasé. Les valeurs obtenues dans le système hexagonal sont :

$$
a=3.194(2) \AA \quad c=4.676(3) \AA
$$

Cette oxydation de $\beta-\mathrm{Co}(\mathrm{OH})_{2}$ par l'oxygène a été mise en évidence antérieurement (5). L'étude par microscopie électronique montre que la transformation est topotactique. La disposition des 2 phases l'une par rapport à l'autre vérifie la relation géométrique:

\section{$[11.0] \beta-\mathrm{Co}(\mathrm{OH})_{2} / /[11.0] \mathrm{CoOOH}$}

L'étude de la thermolyse de l'hydroxyde de cobalt a été réalisée sous azote et sous oxygène. Les résultats présentés sont relatifs au 3ème hydroxyde du tableau I qui contient la plus forte proportion de $\mathrm{CoOOH}(\mathrm{n}=2.37)$. La figure 3 représente les thermogrammes réalisés respectivement : (1) sous azote; 2 sous oxygène. La vitesse de montée en température était de $300^{\circ} \mathrm{C} /$ heure.

(1) Sous azote on observe deux pics endothermiques situés à $170^{\circ} \mathrm{C}$ et $235^{\circ} \mathrm{C}$ environ. Les diagrammes de diffraction $\mathrm{X}$ montrent que le premier pic correspond à la décomposition de $\mathrm{CoOOH}$ en $\mathrm{Co}_{3} \mathrm{O}_{4}$ selon la réaction :

$$
6 \mathrm{CoOOH} \longrightarrow 2 \mathrm{Co}_{3} \mathrm{O}_{4}+1 / 2 \mathrm{O}_{2}+3 \mathrm{H}_{2} \mathrm{O}
$$

La décomposition de l'hydroxyde est à l'origine du 2ème pic, il se produit la réaction:

$$
\mathrm{Co}(\mathrm{OH})_{2} \longrightarrow \mathrm{CoO}+\mathrm{H}_{2} \mathrm{O}
$$

Il est possible de préparer facilement un monoxyde de cobalt de haute pureté par thermolyse sous gaz inerte de $\mathrm{Co}(\mathrm{OH})_{2}$ exempt de $\mathrm{CoOOH}$. Pour éliminer $\mathrm{CoOOH}$ il faut éviter de laisser l'hydroxyde formé au contact de l'oxygène pendant la synthèse.

(2) Sous oxygène on observe un pic exothermique à $150^{\circ} \mathrm{C}$ environ et un pic endothermique à $300^{\circ} \mathrm{C}$. Le pic exothermique correspond à l'oxydation de $\mathrm{Co}(\mathrm{OH})_{2}$ en $\mathrm{Co}_{3} \mathrm{O}_{4}$ selon la réaction:

$$
3 \mathrm{Co}(\mathrm{OH})_{2}+1 / 2 \mathrm{O}_{2} \longrightarrow \mathrm{Co}_{3} \mathrm{O}_{4}+3 \mathrm{H}_{2} \mathrm{O}
$$

Le pic endothermique correspond à la décomposition de $\mathrm{CoOOH}$ en $\mathrm{Co}_{3} \mathrm{O}_{4}$. Conformément à la prévision thermodynamique, la décomposition s'effectue à température plus élevée sous oxygène que sous azote, puisque l'oxygène est un produit de la réaction.

$\underline{\text { B - Hydroxyde de Cadmium }}$

L'oxydation du cadmium dans les conditions opératoires utilisées conduit à $\beta-\mathrm{Cd}(\mathrm{OH})_{2}$. L'analyse chimique d'un échantillon a donné les résultats suivants :

$$
\text { mesuré } \mathrm{Cd} \%=76.1 \quad \text { calculé } \mathrm{Cd} \%=76.77
$$


Le diagramme de diffraction $\mathrm{X}$ a été indexé et les paramètres de la maille hexagonale calculés; l'affinement a donné les résultats suivants :

$$
a=3.496(3) \AA \quad c=4.706(3) \AA
$$

Un cliché de cet hydroxyde réalisé au microscope électronique à balayage haute résolution montre que les cristaux sont parfois fortement agglomérés, ils sont alors de formes très irrégulières. Des formes apparentées à l'hexagone apparaissent pour les cristaux les moins agglomérés. Les dimensions varient de 1 à 5 micromètres environ.

\section{C - Hydroxyde de Nickel}

L'analyse chimique d'un échantillon préparé selon le même mode opératoire que $\mathrm{Co}(\mathrm{OH})_{2}$ a donné les résultats suivants :

$$
\text { mesuré } \mathrm{Ni} \%=62,8 \quad \text { calculé } \mathrm{Ni} \%=63,31
$$

Le diagramme de diffraction X (Fig.4) a été indexé et les paramètres de la maille hexagonale calculés, l'affinement a donné les résultats suivants :

$$
a=3.128(3) \AA \quad c=4.605(5) \AA
$$

La figure 5 représente un cliché de cet hydroxyde réalisé au microscope électronique à balayage haute résolution. L'hydroxyde se présente sous forme de plaquettes approximativement triangulaires plus ou moins agglomérées. Les cotés des triangles ont une dimension moyenne de un micromètre environ.

Les essais réalisés dans le réacteur Buschi ont permis de préciser les facteurs cinétiques inhibant les réactions chimiques. Le blocage de la réaction d'oxydation du nickel à $20^{\circ} \mathrm{C}$ est favorisé par une élévation de la pression partielle d'oxygène. Une élévation ultérieure modérée de la température ne permet pas de faire démarrer la réaction. On a, à titre d'exemple, réalisé les essais suivants :

A la température de $20^{\circ} \mathrm{C}, 30 \mathrm{~g}$ de nickel Inco 255 ont été placés dans le réacteur, puis on a ajouté $300 \mathrm{~cm}^{3}$ d'ammoniaque à $32 \%$. La pression partielle d'ammoniac calculée à partir du titre en $\mathrm{NH}_{3}$ est dans ces conditions de 0.6 bar. La pression totale a été montée jusqu'à 2 bar absolus par de l'oxygène, la pression partielle d'oxygène étant de 1.4 bar. Dans ces conditions le nickel n'est pratiquement pas oxydé. La température a alors été augmentée jusqu'à $40^{\circ} \mathrm{C}$, la pression totale atteignant 3.2 bar: on n'a pas observé de progression de l'oxydation. Ce blocage de la réaction a été attribué à la formation d'une couche passivante d'oxyde de nickel.

Pour vérifier cette hypothèse, le nickel a été récupéré par filtration, lavé, séché puis placé pendant 5 heures à $300^{\circ} \mathrm{C}$ sous un courant d'azote à $10 \%$ d'hydrogène. Aussitôt refroidi il a été introduit dans le réacteur Buschi avec $300 \mathrm{~cm}^{3}$ d'ammoniaque. Les conditions opératoires étaient les suivantes :température $11^{\circ} \mathrm{C}$, pression partielle de $\mathrm{NH}^{3} 0.5$ bar, d'oxygène 0.6 bar.

Des dosages de nickel effectués au bout de $48 \mathrm{~h}$. ont montré que :

$19 \%$ du nickel est dissous sous forme de complexe,

$60 \%$ du nickel est présent sous forme d'hydroxyde ;

$21 \%$ reste sous forme métallique.

L'oxydation se poursuit ensuite plus lentement, car on ne bénéficie pas dans le réacteur Buschi de la séparation magnétique du nickel qui se produit dans le montage de la figure 1.

\section{Conclusion}

Les hydroxydes de cobalt, nickel et cadmium ont été préparés par oxydation du métal par l'oxygène en présence d'ammoniaque. Les synthèses ont été réalisées par réactions à la température ambiante. Les hydroxydes obtenus sont exempts des contaminations inévitables lorsque la méthode classique de précipitation est utilisée.

L'hydroxyde de cobalt doit être récupéré rapidement pour éviter une oxydation ultérieure en $\mathrm{CoOOH}$ par l'oxygène.

La synthèse de l'hydroxyde de nickel nécessite parfois une activation préalable du métal. Cette activation a été réalisée par un traitement de la poudre métallique par l'hydrogène. 
Ces hydroxydes sont des réactifs intéressants pour mener à bien, à basse température, des synthèses d'oxydes simples ou mixtes de haute pureté. La thermolyse de $\mathrm{Co}(\mathrm{OH})_{2}$ en atmosphère neutre est par exemple une méthode simple de préparation du monoxyde de cobalt pur.

\section{Remerciements}

Les auteurs remerciant P. Bernard SAFT Recherche pour la réalisation des clichés M.E.B.

\section{Références}

(1) P. Pascal Nouveau traité de chimie minérale T. XVII. Masson éditeur, 1963 p. 85 et p. 654.

(2) P. Pascal Nouveau traité de chimie minérale T. V. Masson éditeur, 1962 p. 369.

(3) Yichong Guan et Kenneth N. Han. J. Electrochem. Soc. Vol 141 N$^{\circ} 1$ p. 91, (1994).

(4) V.A. Ettel, J. Babjak, S.J. Baksa, D. Shoreline. European patent application number 90104985.8

(5) A. Ammann, W. Feitknecht et R. Giovanoli. 7ème congrès international de microscopie électronique, Grenoble (1970).

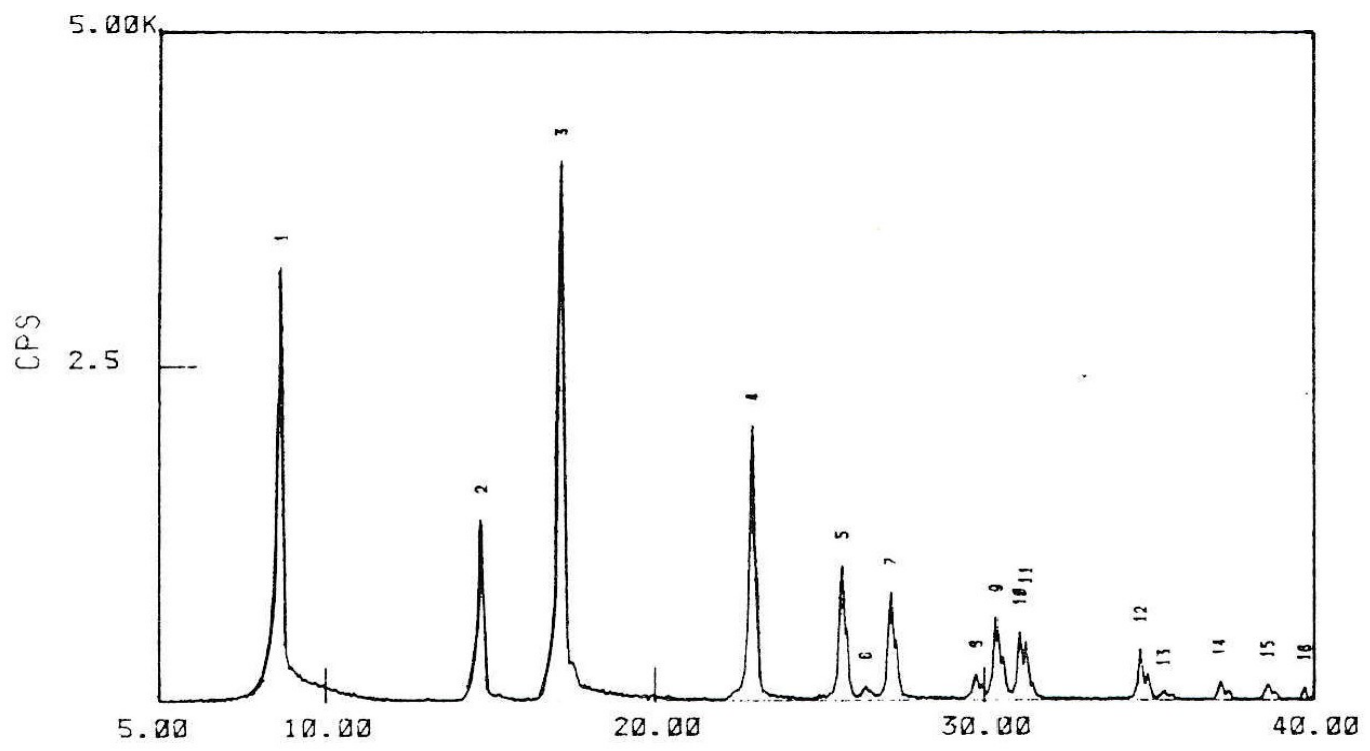

FIG.2 Diffractogramme de $\mathrm{Co}(\mathrm{OH})_{2}$ 


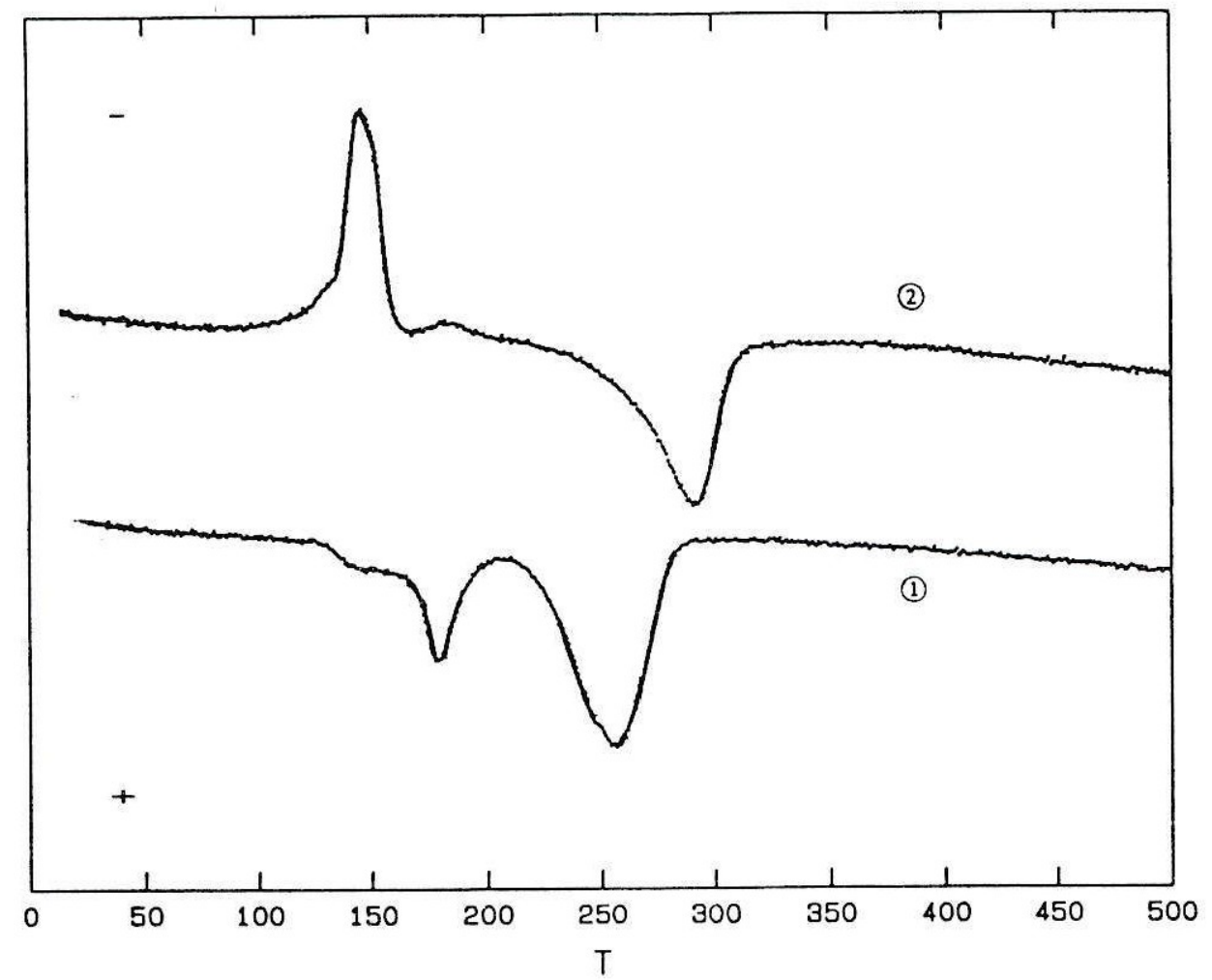

FIG.3

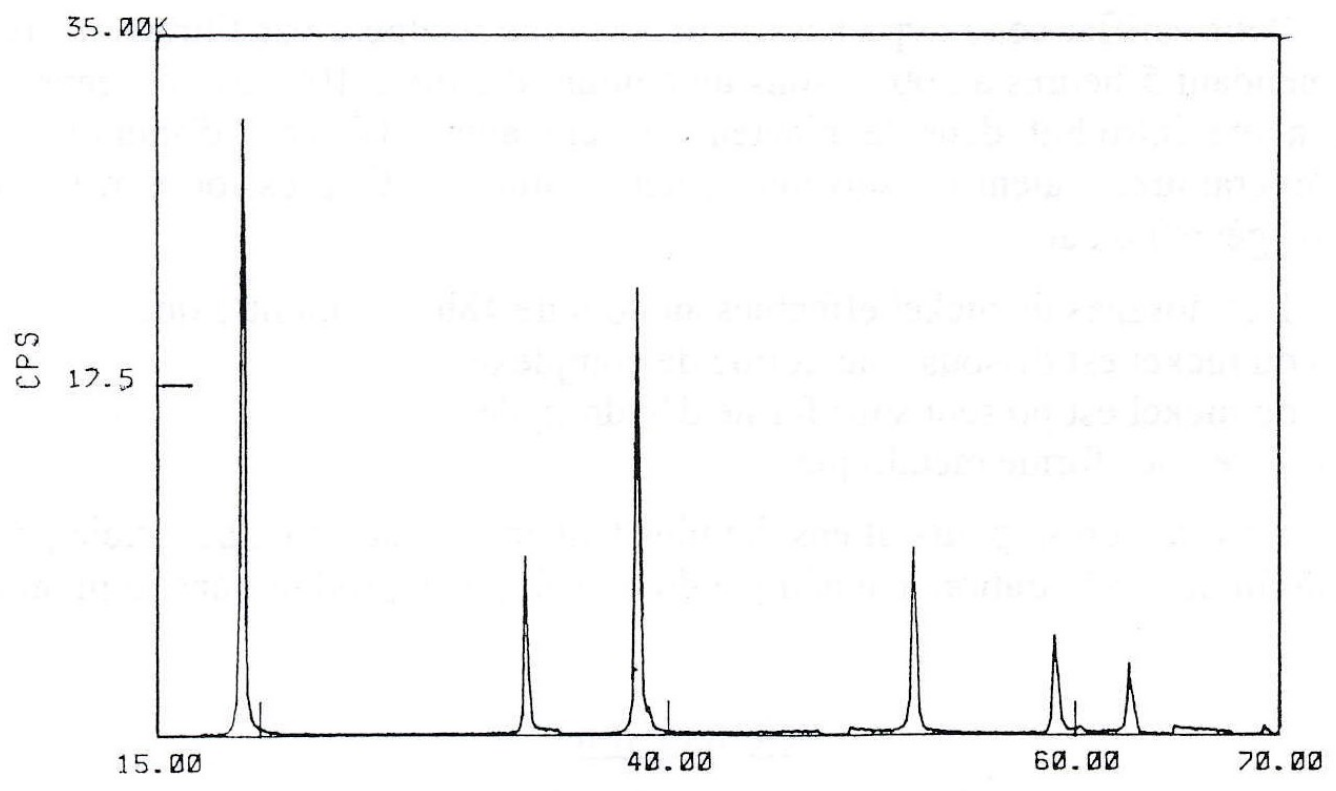

FIG.4 Diffractogramme de $\mathrm{Ni}(\mathrm{OH})_{2}$ 


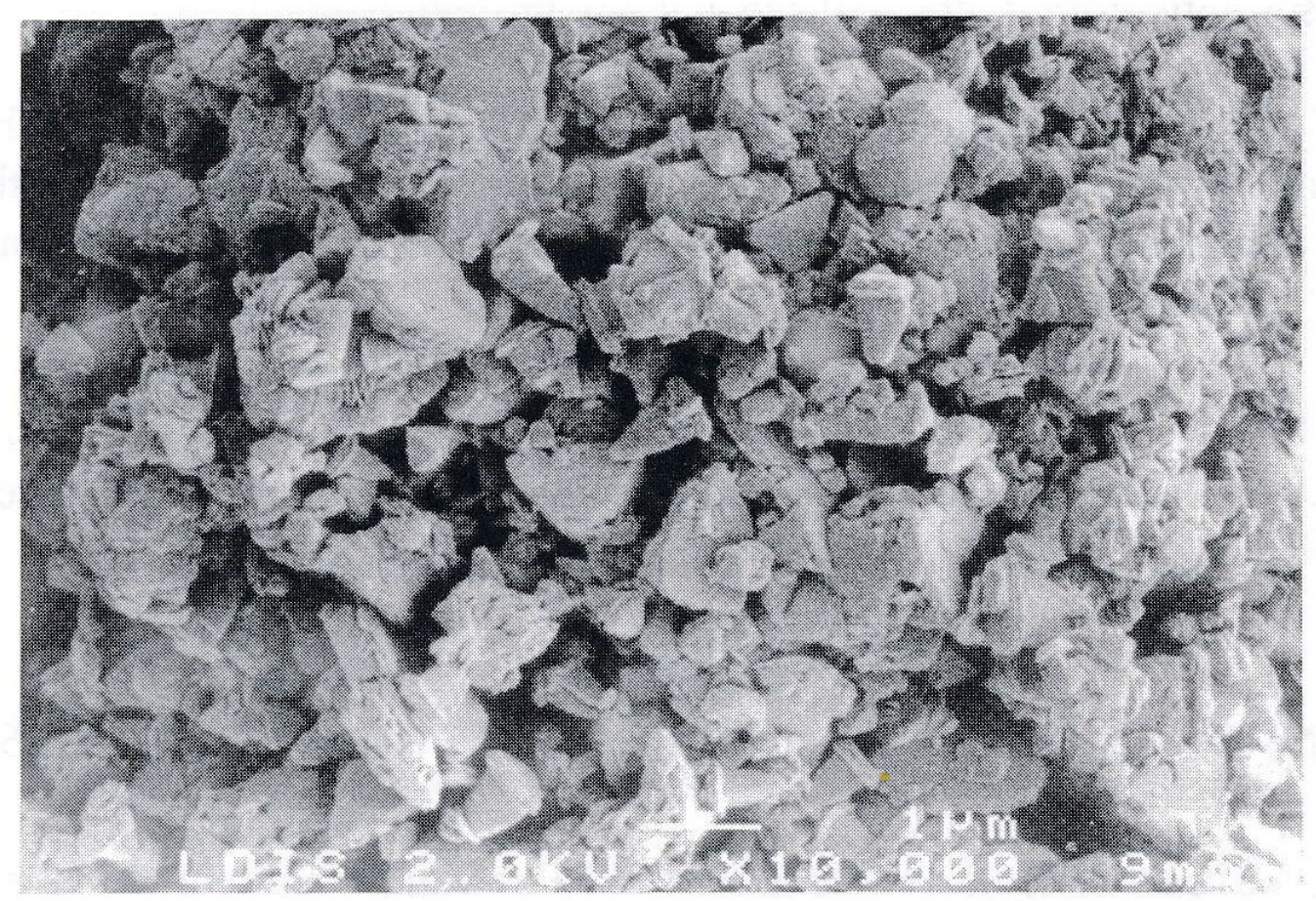

FIG.5 Cliché M.E.B. de $\mathrm{Ni}(\mathrm{OH})_{2}$ 